\title{
Spiking Neuron Model of Basal Forebrain Enhancement of Visual Attention
}

\author{
Michael Avery, Jeffrey L. Krichmar \\ Department of Cognitive Sciences \\ University of California, Irvine \\ Irvine, USA \\ [averym, jkrichma]@uci.edu
}

\author{
Nikil Dutt \\ Department of Computer Science \\ University of California, Irvine \\ Irvine, USA \\ dutt@ics.uci.edu
}

\begin{abstract}
Attentional mechanisms allow the brain to enhance the representation and transmission of certain signals at the expense of others. The basal forebrain has been shown to play an important role in attention through its diverse set of interactions with sensory and associational areas. A recent empirical study indicates that the nucleus basalis, a subset of neurons located in the basal forebrain, is important for improving sensory processing by increasing reliability and decreasing redundancy in the cortex and thalamus $[1,2]$. We developed a spiking neural network model that simulates the nucleus basalis' interaction with the thalamus and visual cortex. In this model, we simulated two modes of action by which it is thought that the nucleus basalis may be influencing sensory processing: (1) inhibitory projections from the nucleus basalis to the thalamic reticular nucleus, which disinhibit the lateral geniculate nucleus (LGN) and gate information into the cortex, and (2) cholinergic excitation of inhibitory neurons in the visual cortex. We showed that the inhibition of the thalamic reticular nucleus GABAergic neurons leads to an increase in the reliability of spikes in the LGN and cortex. We observed that a decrease in the burst to tonic firing ratio in the LGN, coupled with the cholinergic system increasing inhibition in the visual cortex caused decorrelation in the cortex. These findings will help us better understand the mechanisms behind the control of attention by the basal forebrain and shed light on how the orchestrated action of the basal forebrain on multiple target areas can improve information processing in the brain.
\end{abstract}

Keywords - attention; computational neuroscience; microcircuit; neuromodulation; spiking neurons; vision;

\section{INTRODUCTION}

Neuromodulators, such as acetylcholine, signal environmental events, especially under noisy uncertain conditions, and increase attention such that the organism can respond quickly and accurately to these important stimuli [3]. Specifically, attention describes the brain's ability to selectively sharpen or filter sensory information moment by moment. Recent experimental studies have shown that attention causes changes in the variability of neural responses within and between trials [4-6]. The nucleus basalis (NB), a subset of neurons located in the basal forebrain, has been shown to play a key role in attention and arousal through its diverse set of interactions with cortical and subcortical structures [7-9]. It has recently been shown that stimulation of the NB increases neuronal reliability and decreases coding redundancy in the brain by mediating changes in neuronal variability [1]. In their experiment, Goard and Dan recorded spiking activity from all layers of an anaesthetized rat's visual cortex while showing it a video of natural scenes and periodically stimulating the rat's NB. They found that stimulation of the NB increased the between-trial correlation (reliability) and decreased the between-cell correlation (decorrelation) of neurons in visual cortex, which leads to an overall improvement in information processing in the brain [2].

Previous studies have shown that muscarinic receptors (mAChRs) suppress intracortical activity and nicotinic receptors (nAChRs) enhance thalamocortical efficacy $[10,11]$. This led Goard and Dan to hypothesize that (1) mAChRs were responsible for the decorrelation of neuronal signals and (2) $\mathrm{nAChRs}$ were responsible for the increase in reliability. To test (1), they applied a muscarinic antagonist (atropine) to visual cortical neurons while stimulating the NB and saw that the decorrelation decreased, leading them to conclude that muscarinic receptors mediated the active decorrelation of neurons in the cortex. The mechanism behind this increased decorrelation is not completely understood, however, several studies investigating cortical desynchronization and muscarinic receptor distributions help to provide a clearer answer. Muscarinic receptors, for example, have been shown to affect neuronal excitability, synaptic transmission and neuronal plasticity [12] and tend to be localized to inhibitory neurons in visual cortex [13]. It has also been demonstrated that the excitation of inhibitory neurons in a recurrent network may be crucial for decorrelating a population of neurons [14].

Hypothesis (2), however, was refuted when they still measured an increase in response reliability when applying a nicotinic antagonist to the visual cortical neurons. Knowing that the NB modulation of responses must be happening before information gets to the cortex, they then recorded from lateral geniculate nucleus (LGN) neurons and discovered that these neurons also had an increase in reliability with NB stimulation. Since the NB does not project directly to the LGN, it was suggested that the NB's influence on LGN was indirect and that the increase in reliability occurs as a result of the NB disinhibiting the LGN through its GABAergic projections to the thalamic reticular nucleus $[1,2]$. The thalamic reticular nucleus (TRN) is composed of inhibitory neurons and receives topographically organized collaterals from both the cortex and 
the thalamus [15-17]. In turn, the TRN projects to first and higher order thalamic nuclei, including the LGN. The LGN relays all visual sensory information to the cortex and is typically referred to as the gateway to the cortex. Due to its inhibitory projections to LGN, the TRN has been referred to as the "guarding to the gateway" $[18,19]$. The inhibitory projections from the basal forebrain could then regulate the flow of information to the cortex by inhibiting TRN neurons.

We constructed a spiking neuron model of the NB, TRN, LGN, and a V1 cortical column to replicate Goard and Dan's experimental results (Figure 1). Similar to their findings, we were able to show an increase in between-trial correlations in LGN and cortex and a decrease in between-cell correlation in the cortex. Furthermore, we demonstrated that GABAergic projections from the NB to the TRN increased reliability in both the LGN and the cortex, which in turn led to a decrease in the burst to tonic ratio, as was seen in Goard and Dan (2009). We also demonstrated that cholinergic mediated excitation of inhibitory neurons led to a decrease in correlations between neurons in the cortex. These findings will help us better understand the mechanisms behind the control of attention by the basal forebrain and shed light on how the orchestrated action of the basal forebrain on multiple target areas simultaneously can improve information processing in the brain.

\section{METHODS}

\section{A. Stimuli presentation and preprocessing}

Our experiment had a total of 60 trials. In each trial, we presented a 12 second long natural scene video from the van Hateren movie database to the network (http://biology.ucsd.edu/labs/reinagel/pam/NaturalMovie.html). Experiments consisted of 6 blocks of 10 trials. In each block of 10 trials, 5 trials were performed without NB stimulation (control) followed by 5 trials with NB stimulation. In between each trial and block, 1 and 4 seconds, respectively, of random, Poissonian spikes were injected into the network at a rate of 2 $\mathrm{Hz}$ to allow network activity to settle. The total simulation time of the experiment was 13.4 seconds. This took approximately 180 minutes to run on an Intel Core i7 CPU 920 (a) $2.67 \mathrm{GHz}$ with $6 \mathrm{~GB}$ of RAM.

The video contained 300 frames and each frame was presented to the model for $40 \mathrm{~ms}$ of simulation time. Each image was originally $256 \times 256$ pixels. Because our cortical model is a single column, however, the input size was reduced to $20 \times 20$ pixels in order to approximate the visual space that would drive neurons in a single receptive field.

It has been shown that retinal neurons remove linear correlations by "whitening" images before they reach the cortex [20]. To simulate this, all the images were whitened and normalized before being presented to the network. Since we did not want to model the development of orientation selectivity in V1, we assumed that the simulated V1 column was selective to vertical edges. Therefore, the images were convolved with a vertical Gaussian filter after whitening. Inhibitory and excitatory Poisson spike generators converted the images into spike trains in the input layer.

\section{B. Network model}

To develop our model, we used a publicly available simulator, which has been shown to simulate large-scale spiking neural networks efficiently and flexibly [21]. The model contained a subcortical area composed of an input, TRN, LGN, and NB and a four-layered cortical microcircuit (Figure 1). The cortical microcircuit architecture was adapted from [22]. All connections that occur between layers in the microcircuit are shown in Figure 1. Within each layer, there are excitatory-excitatory, excitatory-inhibitory, inhibitoryexcitatory, and inhibitory-inhibitory connections (not shown). Connection probabilities in our cortical model were the same as was used in [22] and can be found in Table 2 of their paper. All subcortical connection probabilities were set to 0.1 except LGN excitatory to L4 excitatory ( $\mathrm{p}=0.15$ ), LGN excitatory to L4 inhibitory $(\mathrm{p}=0.0619)$, and TRN inhibitory to LGN excitatory $(p=0.3)$. The number of neurons in each area is shown in Table 1 below. The model contained a total of 21,903 neurons and approximately 21 million synapses.

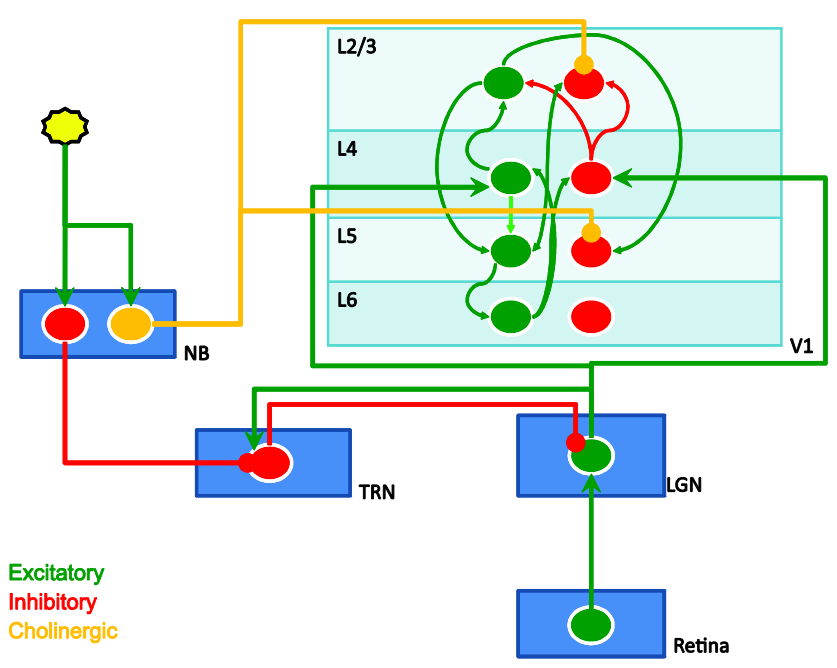

Figure 1: Neural Architecture. The neural network contained an input layer (Retina), 3 subcortical layers (lateral geniculate nucleus (LGN), thalamic reticular nucleus (TRN), nucleus basalis (NB)), and a 4-layer microcircuit (L2/3, L4, L5, L6). Inhibitory, excitatory and cholinergic projections are colored red, green, and yellow, respectively. The retinal group, which is fed input from the video of natural scenes, drives activity in the LGN, which in turn, drives activity in the TRN and L4 of visual cortex. During NBstimulated cases, cholinergic and inhibitory neurons in the NB are excited (indicated by yellow star). Excitation of NB inhibitory neurons results in an inhibition of TRN neurons, disinhibiting the LGN. Excitation of NB cholinergic neurons leads to an increase in the excitability of inhibitory neurons in L2/3 and L5 of the cortex. All connections between layers in the cortical microcircuit are shown. Excitatory-excitatory, excitatory-inhibitory, inhibitory-excitatory, and inhibitory-inhibitory connections within a layer, however, are not shown for clarity. 
TABLE I. NUMBER OF NEURONS PER AREA

\begin{tabular}{|c|c|c|}
\hline Neural Area & Excitatory neurons & Inhibitory neurons \\
\hline Input & 400 & 400 \\
\hline & & \\
\hline Subcortical & & - \\
\hline LGN & 220 & 220 \\
\hline TRN & - & 220 \\
\hline NB & - & 1458 \\
\hline Cortical & & 1369 \\
\hline Layer 2/3 & 5170 & 266 \\
\hline Layer 4 & 5478 & 737 \\
\hline Layer 5 & 1212 & \\
\hline Layer 6 & 3698 & \\
\hline
\end{tabular}

Simple and extended versions of the Izhikevich model were used to govern the dynamics of the spiking neurons in this simulation. Inhibitory and excitatory neurons in the cortex were modeled using the simple Izhikevich model, which are described by the following equations [23]:

$$
\begin{aligned}
& \dot{v}=0.04 v^{2}+5 v+140-u+I \\
& \dot{u}=a(b v-u) \\
& \text { if } v=30, \text { then } v=c, u=u+d
\end{aligned}
$$

where $\mathrm{v}$ is the membrane potential, $\mathrm{u}$ is the recovery variable, I is the input current, and a, b, c, d are parameters chosen based on the neuron type. For regular spiking, excitatory neurons, we set $\mathrm{a}=0.01, \mathrm{~b}=0.2, \mathrm{c}=65.0, \mathrm{~d}=8.0$. For fast-spiking, inhibitory neurons, we set $\mathrm{a}=0.1, \mathrm{~b}=0.2, \mathrm{c}=-65.0, \mathrm{~d}=2.0$. GABAergic and cholinergic neurons in the NB were modeled as simple Izhikevich inhibitory and excitatory neurons, respectively.

LGN and TRN neurons were modeled using the extended version of the Izhikevich neuron model in order to account for the bursting and tonic modes of activity, which these neurons have shown to exhibit [24]. The equations governing these neurons are given as:

$$
\begin{aligned}
& C \dot{v}=k\left(v-v_{r}\right)\left(v-v_{t}\right)-u+I \\
& \dot{u}=a\left[b\left(v-v_{r}\right)-u\right]
\end{aligned}
$$

The equations for this extended model are similar to the previous model, except they include additional parameters, such as: membrane capacitance $(\mathrm{C})$, resting potential $\left(\mathrm{V}_{\mathrm{r}}\right)$, and instantaneous threshold potential $\left(\mathrm{v}_{\mathrm{t}}\right)$. For LGN neurons, parameters were set to: $\mathrm{a}=0.1, \mathrm{c}=-60, \mathrm{~d}=10, \mathrm{C}=200, \mathrm{v}_{\mathrm{r}}=60$, $\mathrm{v}_{\mathrm{t}}=-50$. For TRN neurons, parameters were set to: $\mathrm{a}=0.015$, $\mathrm{c}=55, \mathrm{~d}=50, \mathrm{C}=40, \mathrm{v}_{\mathrm{r}}=65, \mathrm{v}_{\mathrm{t}}=45$. In order to simulate the switch between bursting (Figure 2A) and tonic (Figure 2B) mode, the $\mathrm{b}$ parameter, which is related to the excitability of the cell, was changed depending upon membrane potential, v. Specifically, if $\mathrm{v}<-65$, b was set equal to 70 and the neuron would be in bursting mode. If $v>-65$, $b$ was set equal to 0 and the neuron would be in tonic mode.

The synaptic input, $I$, driving each neuron was dictated by simulated AMPA, NMDA, GABA ${ }_{A}$ and $\mathrm{GABA}_{\mathrm{B}}$ conductances $[21,24]$. The total synaptic input seen by each neuron was given by:

$$
\begin{gathered}
I=g_{A M P A}(v-0)+g_{N M D A} \frac{\left[\frac{v+80}{60}\right]^{2}}{1+\left[\frac{v+80}{60}\right]^{2}}(v-0) \\
+g_{G A B A_{A}}(v+70)+g_{G A B A_{B}}(v+90)
\end{gathered}
$$

where $v$ is the membrane potential and $\mathrm{g}$ is the conductance. The conductances change according to the following first order equation:

$$
\dot{g}_{i}=-\frac{g}{\tau_{i}}
$$

where $\tau_{\mathrm{i}}=5,100,6,150 \mathrm{~ms}$ for $\mathrm{i}=\mathrm{AMPA}, \mathrm{NMDA}, \mathrm{GABA}_{\mathrm{A}}$, $\mathrm{GABA}_{\mathrm{B}}$ conductances, respectively. When an excitatory (inhibitory) neuron fires $\mathrm{g}_{\mathrm{AMPA}}$ and $\mathrm{g}_{\mathrm{NMDA}}\left(\mathrm{g}_{\mathrm{GABAA}}\right.$ and $\left.\mathrm{g}_{\mathrm{GABAB}}\right)$ increase by the synaptic weight, $w$, between pre- and postsynaptic neurons.
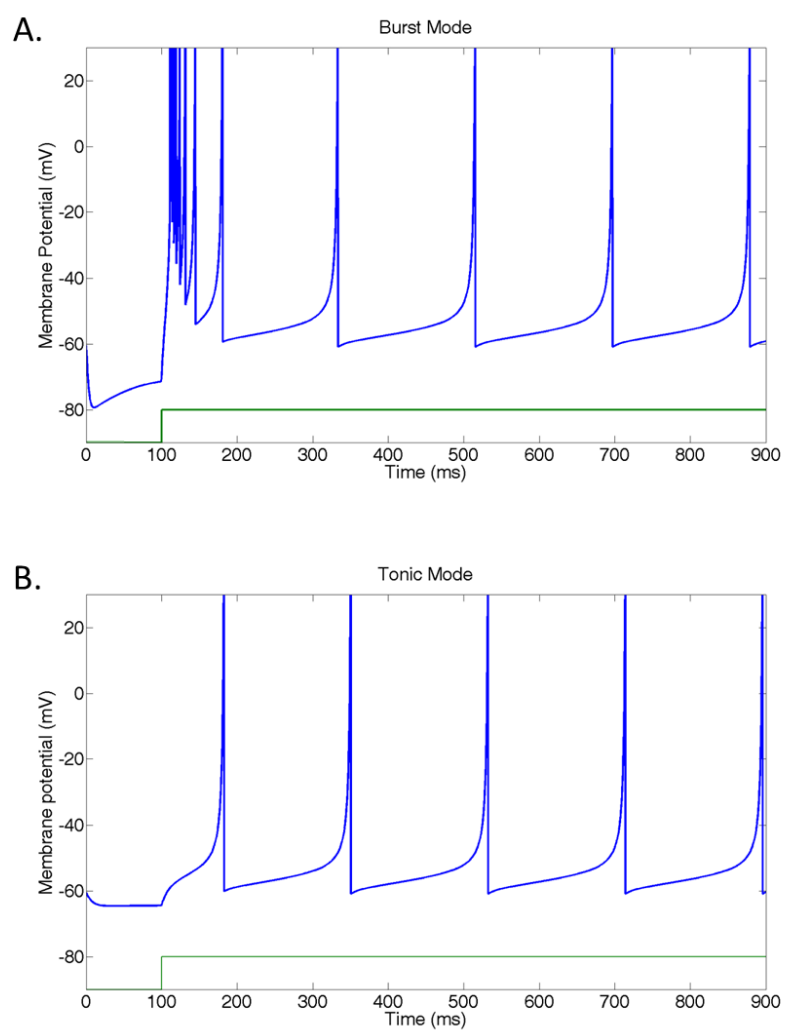

Figure 2: A. Example LGN neuron in bursting mode. The membrane potential was held below $-65 \mathrm{mV}$ (b parameter set equal to 70 , see text) by injecting a strong inhibitory current into the cell until $\mathrm{t}=100 \mathrm{~ms}$ at which time a step excitatory current (green line) was injected into the cell. B. Example LGN neuron in tonic mode. The membrane potential was held at around -60 $\mathrm{mV}$ by injecting a weak inhibitory current into the cell until $\mathrm{t}=100 \mathrm{~ms}$ at which time a step excitatory current (green line) was injected into the cell.

\section{NB modulation of cortical and subcortical structures}

The simulated NB modulated activity in the network in two ways. First, in trials in which the NB was stimulated, excitatory Poisson spike trains drove GABAergic neurons within the NB. These GABAergic neurons projected from the NB to the TRN, inhibiting GABAergic neurons in the TRN. 
This in turn released TRN inhibition of LGN. Second, cholinergic projections from NB to inhibitory neurons in the cortical microcircuits were simulated. It has been shown that mAChRs tend to be localized on inhibitory neurons in the visual cortex and are likely to increase their excitability [13, 25]. When the NB was stimulated, the b parameter in the Izhikevich model, which controls cell excitability, was increased from 0.20 to 0.32 for inhibitory neurons in layers 2 and 5 of the cortical microcircuit. This is intended to mimic the cholinergic activation of mAChRs on inhibitory neurons, which leads to increased cell excitability.

\section{Push-pull}

An important component in our model for generating bursts in the LGN was the push-pull mechanism. It has been suggested that switching between burst and tonic mode is facilitated by a "push-pull" mechanism through feed-forward excitation and inhibition from the retina to the LGN [26, 27]. This mechanism involves input from the retina hyperpolarizing LGN neurons through feed-forward inhibitory circuits when luminance levels are low or zero, and depolarizing LGN neurons through feed-forward excitation after luminance levels reach a certain threshold. We implemented this phenomenon by having inhibitory Poisson neurons driving the LGN when inputs were less than 0 and excitatory Poisson neurons driving the LGN when inputs were greater than 0 .
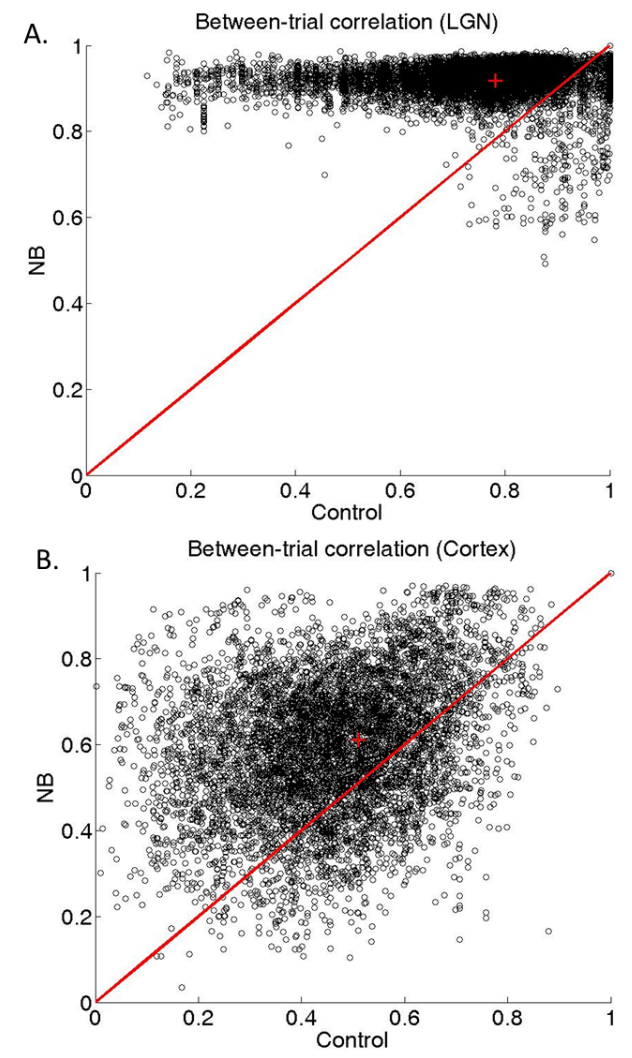

Figure 3: A. Between-trial correlation in LGN. B. Between-trial correlation in cortex (100 neurons in each layer). Scatter plots showing the between-trial correlation of neurons in both the control (x-axis) and NB-stimulated (y-axis) cases. Responses from single neurons were taken and compared across trials.
As can be seen in Figure 3A and 3B, the centroid (indicated by the red "+" symbol) is situated higher on the $\mathrm{y}$-axis in both the LGN (Figure 3A, $\mathrm{x}=0.89, \mathrm{y}=0.78$ ) and the cortex (Figure $3 \mathrm{~B}, \mathrm{x}=0.61, \mathrm{y}=0.5$ ) due to a larger number of scatter points above the line $y=x$. This indicated that during the NB-stimulated case, neurons in both the cortex and LGN have a higher degree of correlation between trials when compared with the control case. This implies that the reliability of neurons tends to increase when the NB is stimulated.

\section{RESULTS}

\section{A. Increase in between-trial correlation}

The between trial correlation was measured in the LGN and the cortex during both control and NB stimulated cases. This was done by first binning the spikes of a single neuron at $25 \mathrm{~Hz}$ and grouping the responses into trials. We then used the MATLAB routine corrcoef to compute the correlation coefficient for each neuron in the LGN across trials in control and NB stimulated cases. In Figure 3, we show that the cortex and LGN both show an increase in between-trial correlation (reliability) when the NB was stimulated versus when the NB is not stimulated (control). Goard and Dan recorded neurons in both the LGN and cortex and showed similar increases in the between-trial correlation of these neurons as a result of NB stimulation (see Figures $4 \mathrm{~b}$ and $6 \mathrm{~b}$ in [1]). In our model, this increase in reliability happens as a result of GABAergic projections from the NB inhibiting inhibitory cells in the TRN, which in turns disinhibits the LGN. We suspect that the differences in the mean and variance of the LGN and cortical plots (Figure 3A and B) occurred as a result of feedforward inhibition from LGN to layer 4 inhibitory neurons, which has been shown reduce correlations [28].

\section{B. Burst-to-tonic ratio}

We also measured the burst-to-tonic ratio in the LGN. A series of spikes are declared a "burst" when they occur with inter-spike intervals less than $4 \mathrm{~ms}$ followed by the absence of a spike for $100 \mathrm{~ms}$ or more. Tonic spikes were any spikes that were not part of a burst. We plotted the ratio of the number of burst spikes to the number of tonic spikes during both the control and NB stimulated cases (Figure 4). As shown in Figure 4, when the NB is stimulated the burst to tonic ratio is smaller than in control conditions, indicating that bursting behavior in the LGN decreases when the NB is stimulated. Goard and Dan recorded neurons in the LGN and showed similar decreases in the burst-to-tonic ratio of these neurons as a result of NB stimulation (see Figure $6 \mathrm{c}$ in [1]). In our model, when inhibitory projections in the NB are stimulated, they hyperpolarize TRN neurons. As a result, TRN neurons are less active and have less inhibitory control over LGN neurons, which depolarizes LGN neurons and switches them from bursting to tonic firing mode. 


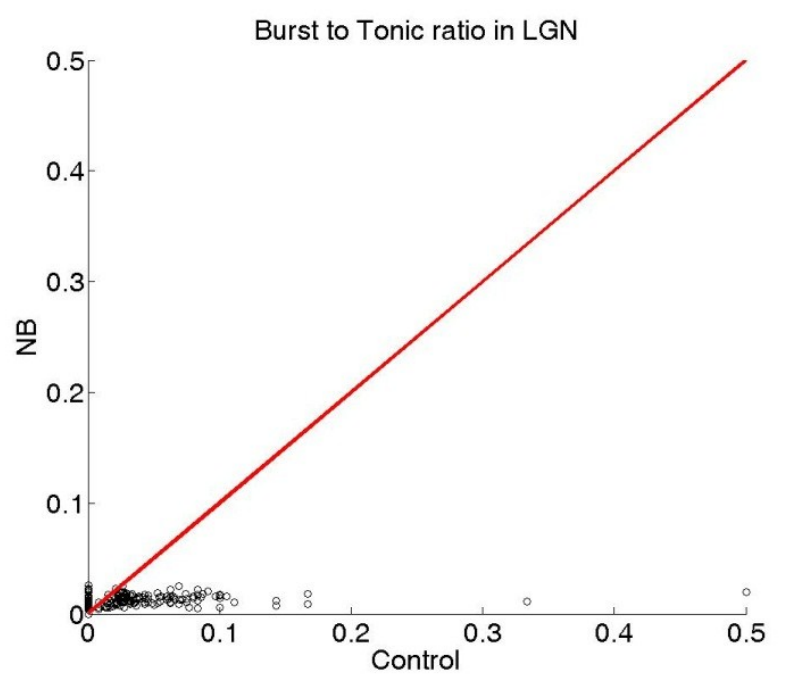

Figure 4: Burst-to-tonic ratio in LGN. Scatterplot showing the burst-to-tonic ratio in the LGN in both control (x-axis) and NB-stimulated (y-axis) cases. This plot shows that when the NB is stimulated the burst-to-tonic ratio is larger compared to the non-NB stimulated (control) condition. This means that the number of burst spikes decreases relative to the number of tonic spikes when the NB is stimulated.

\section{Decrease in between-cell correlation}

Goard and Dan showed that when the NB was stimulated, neurons became decorrelated in the visual cortex. When a $\mathrm{mAChR}$ antagonist was applied, however, the decorrelation decreased. This indicated that mAChRs mediated this decorrelation (see Figures $3 b$ and $5 b$ in [1]). It has been shown that $\mathrm{mAChRs}$ tend to be localized on inhibitory neurons in V1 [12] and, when activated, excite the cell upon which they are located [24]. Thus, in our simulation, when NB was stimulated we increased the excitability of V1 inhibitory interneurons in layers 2 and 5 by changing the $\mathrm{b}$ parameter from 0.20 to 0.32 . In Figure 5A and 5B, we show scatter plots of the between-cell correlation with and without the mAChR-mediated excitation of inhibitory neurons in V1, respectively. The plot of betweencell correlation without $\mathrm{mAChRs}$ was meant to simulate the application of a mAChR antagonist as was shown in Figure $5 \mathrm{~b}$ of [1]. In each plot, the NB stimulated case was plotted on the $\mathrm{y}$-axis and the non-NB stimulated (control) case was plotted on the x-axis. The centroids (indicated by the red "+" symbol) indicate where the center of mass is for the set of scatter points in each figure. A lower (higher) center of mass in the ydirection implies that NB stimulation led to a decrease (increase) in correlations. The centroid in the models that were run with simulated mAChRs is centered at $(0.89,0.61)$. The centroid in models that were run without simulated mAChRs (simulating the application of a muscarinic antagonist) was centered at $(0.89,0.78)$. This implies that the addition of simulated mAChRs to the model (i.e. excitation of L2/3 and L5 inhibitory neurons) was necessary for increasing decorrelation amongst neurons and removing the simulated mAChRs (i.e. simulating the application of a muscarinic antagonist) removes this decorrelation.
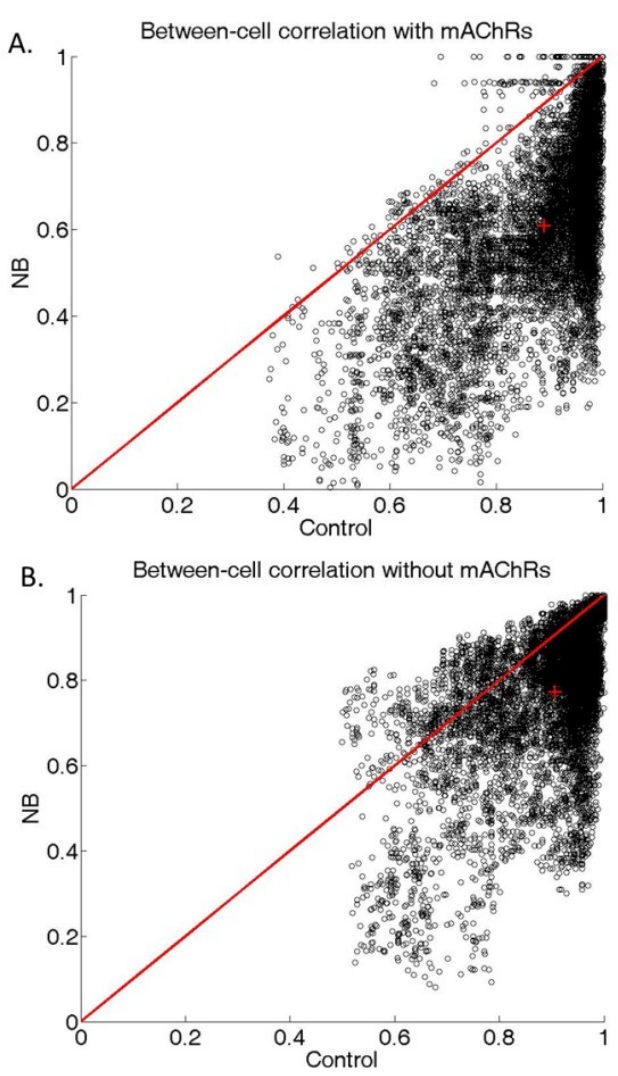

Figure 5: A. Between-cell correlation in cortex with $\mathrm{mAChR}$. B. Betweencell correlation in cortex without $\mathrm{mAChR}$. Scatter plots showing the betweencell correlation of neurons (100 neurons from each layer) in the cortex in both the control (x-axis) and NB-stimulated (y-axis) cases. As can be seen in comparing Figure 5A and 5B, the centroid (indicated by the red "+" symbol) is situated lower on the $\mathrm{y}$-axis when simulated $\mathrm{mAChRs}$ are present (Figure $5 \mathrm{~A}, \mathrm{x}=0.89, \mathrm{y}=0.61$ ) than when they are not present (Figure 5B, $\mathrm{x}=0.89, \mathrm{y}=0.78$ ) due to a larger number of scatter points below the line $y=x$. This means, that when the simulated $\mathrm{mAChRs}$ are present (i.e. inhibitory neurons in L2/3 and L5 are excited), there is less between-cell correlation (i.e. decorrelation) between neurons in the cortex. This implies that excitation of inhibitory neurons via the activation of mAChRs is a mechanism by which the cortex actively decorrelates incoming inputs.

\section{DISCUSSION}

In this paper, we presented a spiking neuron model of the basal forebrain, thalamus, and visual cortex that matched experimentally verified data, and demonstrated how the nucleus basalis of the basal forebrain increases neuronal reliability and decreases between-cell correlation in the brain. We demonstrated possible pathways and cellular mechanisms by which these changes in neuronal dynamics may be occurring. First, we showed that NB stimulation in our model led to an increase in reliability in the LGN and cortex and a decrease in the burst-to-tonic ratio in the LGN, as a result of the activation of GABAergic projections from the NB to the TRN. This finding adds evidence to the notion that inhibition of the TRN by NB is a means by which the NB can switch LGN cells from bursting to tonic mode. This switch in firing mode leads to an increase in neuronal reliability in LGN and cortex. Our simulations demonstrated a mAChR mediated mechanism for the decrease in between-cell correlation in the cortex that occurs with NB stimulation. In our model, the 
mAChR mediated decorrelation of neuronal responses in the cortex happened as a result of excitation of fast-spiking inhibitory neurons. This suggests that the activation of inhibitory neurons via neuromodulators in cortex is a possible mechanism by which the cortex actively decorrelates its input in order to decrease redundancy.

Goard and Dan proposed that the increase in reliability resulted from a switch in LGN neurons from bursting to tonic mode. Bursting mode occurs when a cell is hyperpolarized to the extent that it leads to a low threshold $\mathrm{Ca}^{2+}$ spike that triggers a burst in action potentials [29]. In tonic mode, the cell is depolarized and linearly sums its input. As a result, when LGN cells are in tonic mode they will more reliably send the signal to the cortex, whereas in burst mode the detectability of the signal is greater at the expense of a decrease in reliability. Since the NB does not directly project to the LGN, it was suggested that inhibitory neurons in the NB may inhibit GABAergic neurons in the TRN, leading to a depolarization of LGN cells. We tested the plausibility of this prediction by modeling the NB to TRN pathway and found that stimulation of the NB could lead to increased reliability in the cortex via a switch from bursting to tonic firing.

Changing the degree of correlation in a group of neurons has been shown to be an important mechanism involved in attention [4-6, 30]. Our model suggests that mAChRs mediate the decorrelation of neuronal responses in visual cortex via cholinergic excitation of fast-spiking inhibitory neurons. The biophysical mechanisms involved in modulating correlations are not completely understood, however, several studies suggest it may involve inhibitory neurons [6, 14, 28, 31], synaptic depression [32], and/or slow afterhyperpolarization currents [33]. Our result matches well with theoretical and computational studies which highlight the importance of inhibition in decorrelating neurons [14], and experimental studies, which show that mAChRs are typically expressed on fast-spiking inhibitory neurons [13] and increase neuronal excitability [25].

Several computational models have been recently developed that show how neuromodulation can effect cortical processing. The SMART model (Synchronous Matching Adaptive Resonance Theory), developed by Grossberg and Versace [34], is a spiking model that includes a detailed cortical and subcortical (thalamic) circuit design as well as synaptic plasticity and cholinergic neuromodulation. This model shows how top-down expectations and bottom-up input may be compared and might lead to changes in plasticity. Specifically, if top-down and bottom-up information match (resonate), learning is increased, whereas, if top-down and bottom-up input mismatch, a reset state is triggered and learning is reduced. The cholinergic system in this model is associated with the level of vigilance and regulates the degree by which top-down and bottom-up input must match in order to trigger states of learning or reset. Deco and Thiele [35] also developed a model demonstrating how cholinergic activity effects the interaction between top-down attentional input and bottom-up sensory information in a cortical area. Their model incorporated four ways in which the cholinergic system has been shown to influence the cortex, including: (1) reducing firing rate adaptation, (2) increasing thalamocortical efficacy,
(3) reducing lateral interactions, and (4) increasing inhibitory drive. They showed that the dominant way in which the cholinergic system effects top-down attentional modulation is by reducing lateral interactions amongst neurons in the cortex. Finally, a third model was developed that demonstrates how the cholinergic and noradrenergic systems track expected and unexpected uncertainty in the environment, respectively, and effect several cortical targets in order to optimize behavior [36]. This model incorporated five different ways in which the cholinergic and noradrenergic systems may influence cortical processing, including: (1) nicotinic enhancement of thalamocortical input, (2) muscarinic regulation of corticocortical feedback, (3) noradrenergic mediation of a network reset, (4) locus coeruleus (LC) activation of the basal forebrain (BF), and (5) cholinergic and noradrenergic balance between sensory input and frontal cortex predictions. The simulation was able to make several interesting predictions, including how the BF and LC influence one another and how faults in either system can lead to deficits in cortical processing and degradation of behavior.

The present model differs from those mentioned above in a few important ways. First, we showed how non-cholinergic neurons (GABAergic) in the basal forebrain can influence subcortical structures (TRN). The three papers above, on the other hand, concentrated exclusively on cholinergic neurons in the $\mathrm{BF}$ and their influence on the cortex. Our model also focuses on reproducing the experimental results found by Goard and Dan given current knowledge about muscarinic receptor distributions and their influence on cell excitability. This allowed us to come to the more specific conclusion that cholinergic modulation alters information processing in visual cortex via mAChR-mediated increase in the excitability of inhibitory neurons in layers 2 and 5 . Finally, because we developed a microcircuit model, our model adds a level of detail in terms of circuit architecture that is missing in [35] and [36]. The SMART model on the other hand, has a highly detailed microcircuit architecture. In the future it would be interesting to combine the level of detail of our model and the SMART model with the wide range of cholinergic actions that were incorporated into [35] and [36].

In both the Goard and Dan experiment and our simulation the NB was artificially stimulated, however, it is important to keep in mind what drives the NB in order to better understand its role in the brain. It has been suggested that the basal forebrain responds to the amount of expected uncertainty in the environment [37]. Strong projections from association, sensory, and subcortical brain regions drive the NB, indicating that the NB plays an important role in balancing bottom-up (sensory) and top-down (expectations) information [9]. This suggests that when there is a large amount of uncertainty in either expectations or sensory input, the basal forebrain may be recruited to coordinate changes in information processing in multiple areas across the brain.

In addition to its role in sensory processing, the basal forebrain has also been shown to play an important role in working memory [38], attention [39], and the encoding of longterm memories [40] via its projections to prefrontal, parietal, and hippocampal cortices. This suggests that when there is a large amount of uncertainty in either your priors or sensory 
input, the basal forebrain may be recruited to coordinate changes in information processing in multiple areas across the brain in order to ultimately optimize behavior. In order to truly understand the link between attention, learning, memory, and action-selection in the brain, it will be important to further elucidate how and when the basal forebrain is activated and the effect of basal forebrain's impact on cortical and subcortical targets.

\section{ACKNOWLEDGEMENT}

We thank Y. Dan for providing helpful details behind the experimental results that served as a basis for our model. This work was supported by the Defense Advanced Research Projects Agency (DARPA) subcontract 801888-BS, and NSF award number IIS-0910710.

\section{REFERENCES}

[1] M. Goard and Y. Dan, "Basal forebrain activation enhances cortical coding of natural scenes," Nat Neurosci, vol. 12, pp. 1444-9, Nov 2009.

[2] A. Thiele, "Optimizing brain processing," Nat Neurosci, vol. 12, pp. 1359-60, Nov 2009.

[3] J. Krichmar, "The neuromodulatory system: a framework for survival and adaptive behavior in a challenging world," Adaptive Behavior, vol. 16, pp. 385-399, 2008.

[4] M. R. Cohen and J. H. Maunsell, "Attention improves performance primarily by reducing interneuronal correlations," Nat Neurosci, vol. 12, pp. 1594-600, Dec 2009.

[5] J. F. Mitchell, K. A. Sundberg, and J. H. Reynolds, "Spatial attention decorrelates intrinsic activity fluctuations in macaque area V4," Neuron, vol. 63, pp. 879-88, Sep 242009.

[6] K. D. Harris and A. Thiele, "Cortical state and attention," Nat Rev Neurosci, vol. 12, pp. 509-23, Sep 2011.

[7] I. Klinkenberg, A. Sambeth, and A. Blokland, "Acetylcholine and attention," Behav Brain Res, vol. 221, pp. 430-42, Aug 102011.

[8] B. Noudoost and T. Moore, "The role of neuromodulators in selective attention," Trends Cogn Sci, vol. 15, pp. 585-91, Dec 2011.

[9] M. Sarter, M. E. Hasselmo, J. P. Bruno, and B. Givens, "Unraveling the attentional functions of cortical cholinergic inputs: interactions between signal-driven and cognitive modulation of signal detection," Brain Res Brain Res Rev, vol. 48, pp. 98111, Feb 2005.

[10] A. A. Disney, C. Aoki, and M. J. Hawken, "Gain modulation by nicotine in macaque v1," Neuron, vol. 56, pp. 701-13, Nov 212007.

[11] M. E. Hasselmo and J. McGaughy, "High acetylcholine levels set circuit dynamics for attention and encoding and low acetylcholine levels set dynamics for consolidation," Prog Brain Res, vol. 145 , pp. 207-31, 2004.
[12] N. Origlia, N. Kuczewski, E. Pesavento, E. Aztiria, and L. Domenici, "The role of cholinergic system in neuronal plasticity: focus on visual cortex and muscarinic receptors," Arch Ital Biol, vol. 146, pp. 165-88, Sep 2008.

[13] A. A. Disney, K. V. Domakonda, and C. Aoki, "Differential expression of muscarinic acetylcholine receptors across excitatory and inhibitory cells in visual cortical areas V1 and V2 of the macaque monkey," J Comp Neurol, vol. 499, pp. 49-63, Nov 1 2006.

[14] A. Renart, J. de la Rocha, P. Bartho, L. Hollender, N. Parga, A. Reyes, and K. D. Harris, "The asynchronous state in cortical circuits," Science, vol. 327, pp. 587-90, Jan 292010.

[15] Y. B. Saalmann and S. Kastner, "Cognitive and perceptual functions of the visual thalamus," Neuron, vol. 71, pp. 209-23, Jul 282011.

[16] D. Pinault, "The thalamic reticular nucleus: structure, function and concept," Brain Res Brain Res Rev, vol. 46, pp. 1-31, Aug 2004.

[17] K. McAlonan, J. Cavanaugh, and R. H. Wurtz, "Attentional modulation of thalamic reticular neurons," J Neurosci, vol. 26, pp. 4444-50, Apr 19 2006.

[18] K. McAlonan, J. Cavanaugh, and R. H. Wurtz, "Guarding the gateway to cortex with attention in visual thalamus," Nature, vol. 456, pp. 391-4, Nov 20 2008.

[19] F. Crick, "Function of the thalamic reticular complex: the searchlight hypothesis," Proc Natl Acad Sci U S $A$, vol. 81, pp. 4586-90, Jul 1984.

[20] E. P. Simoncelli and B. A. Olshausen, "Natural image statistics and neural representation," Annu Rev Neurosci, vol. 24, pp. 1193-216, 2001.

[21] M. Richert, J. M. Nageswaran, N. Dutt, and J. L. Krichmar, "An efficient simulation environment for modeling large-scale cortical processing," Front Neuroinform, vol. 5, p. 19, 2011.

[22] N. Wagatsuma, T. C. Potjans, M. Diesmann, and T. Fukai, "Layer-Dependent Attentional Processing by Top-down Signals in a Visual Cortical Microcircuit Model," Front Comput Neurosci, vol. 5, p. 31, 2011.

[23] E. M. Izhikevich, "Simple model of spiking neurons," IEEE Trans Neural Netw, vol. 14, pp. 1569-72, 2003.

[24] E. M. Izhikevich and G. M. Edelman, "Large-scale model of mammalian thalamocortical systems," Proc Natl Acad Sci US A, vol. 105, pp. 3593-8, Mar 4 2008.

[25] D. A. McCormick and D. A. Prince, "Mechanisms of action of acetylcholine in the guinea-pig cerebral cortex in vitro," J Physiol, vol. 375, pp. 169-94, Jun 1986.

[26] X. Wang, F. T. Sommer, and J. A. Hirsch, "Inhibitory circuits for visual processing in thalamus," Curr Opin Neurobiol, Jul 112011. 
[27] X. Wang, Y. Wei, V. Vaingankar, Q. Wang, K. Koepsell, F. T. Sommer, and J. A. Hirsch, "Feedforward excitation and inhibition evoke dual modes of firing in the cat's visual thalamus during naturalistic viewing," Neuron, vol. 55, pp. 465-78, Aug 22007.

[28] J. W. Middleton, C. Omar, B. Doiron, and D. J. Simons, "Neural correlation is stimulus modulated by feedforward inhibitory circuitry," J Neurosci, vol. 32, pp. 506-18, Jan 112012.

[29] S. M. Sherman, "Dual response modes in lateral geniculate neurons: mechanisms and functions," Vis Neurosci, vol. 13, pp. 205-13, Mar-Apr 1996.

[30] P. Fries, J. H. Reynolds, A. E. Rorie, and R. Desimone, "Modulation of oscillatory neuronal synchronization by selective visual attention," Science, vol. 291, pp. 1560-3, Feb 232001.

[31] D. Battaglia, N. Brunel, and D. Hansel, "Temporal decorrelation of collective oscillations in neural networks with local inhibition and long-range excitation," Phys Rev Lett, vol. 99, p. 238106, Dec 7 2007.

[32] M. S. Goldman, S. B. Nelson, and L. F. Abbott, "Decorrelation of spike trains by synaptic depression," Neurocomputing, vol. 26-27, p. 7, 1999.

[33] X. J. Wang, Y. Liu, M. V. Sanchez-Vives, and D. A. McCormick, "Adaptation and temporal decorrelation by single neurons in the primary visual cortex," $J$ Neurophysiol, vol. 89, pp. 3279-93, Jun 2003.

[34] S. Grossberg and M. Versace, "Spikes, synchrony, and attentive learning by laminar thalamocortical circuits," Brain Res, vol. 1218, pp. 278-312, Jul 7 2008.

[35] G. Deco and A. Thiele, "Cholinergic control of cortical network interactions enables feedbackmediated attentional modulation," Eur J Neurosci, vol. 34, pp. 146-57, Jul 2011.

[36] M. C. Avery, D. A. Nitz, A. A. Chiba, and J. L. Krichmar, "Simulation of cholinergic and noradrenergic modulation of behavior in uncertain environments," Front Comput Neurosci, vol. 6, p. 5, 2012.

[37] A. J. Yu and P. Dayan, "Uncertainty, neuromodulation, and attention," Neuron, vol. 46, pp. 681-92, May 192005.

[38] P. L. Croxson, D. A. Kyriazis, and M. G. Baxter, "Cholinergic modulation of a specific memory function of prefrontal cortex," Nat Neurosci, vol. 14, pp. 1510-2, 2011.

[39] J. I. Broussard, K. Karelina, M. Sarter, and B. Givens, "Cholinergic optimization of cue-evoked parietal activity during challenged attentional performance," Eur J Neurosci, vol. 29, pp. 1711-22, Apr 2009.

[40] M. E. Hasselmo, "Neuromodulation: acetylcholine and memory consolidation," Trends Cogn Sci, vol. 3, pp. 351-359, Sep 1999. 